\title{
pH-responsive mesoporous silica nanocarriers based on layer-by-layer self- assembly
}

\author{
Hong-Yu Zhang*, Yan-Fang Sun, Yu-Long Sun and Ming Zhou \\ State Key Laboratory of Tribology, Department of Mechanical Engineering, Tsinghua University, \\ Beijing 100084, China
}

\begin{abstract}
Significant progress has been achieved in the field of pH-sensitive drug delivery system in recent years. For layerby-layer self-assembly nanocarriers, various $\mathrm{pH}$ values can induce the change of permeability and structural stability of the outer polyelectrolyte multi-layers, resulting in the release of the loaded drug. In the present study, carboxyl-modified mesoporous silica nanoparticles (MSNs-COOH) were prepared as reservoirs to hold model cargo, i.e. Rhodamine B (RhB). Polycations, including 1, 4-butanediamine (BDA)-modified linear poly (glycerol methacrylate) (L-B) or BDA-modified starshaped poly (glycerol methacrylate) (S5-B) and 1,2-ethanediamine (EDA)-modified star-shaped poly(glycerol methacrylate) (S5-E), and polyanion, i.e. poly(acrylic acid) (PAA), are self-assembled by electrostatic interactions as coating. The loading process of RhB was characterized by transmission electron microscopy, Fourier transform infrared spectroscopy, thermogravimetric analysis, and dynamic light scattering, and the release of $\mathrm{RhB}$ was controlled by changing the $\mathrm{pH}$ of phosphate buffer saline. The results showed that the loading capacity and entrapment efficiency of RhB were the highest for S5-B/PAA, which were $10.1 \%$ and $44.4 \%$, respectively. In addition, the release of $\mathrm{RhB}$ was about $90 \%$ at $\mathrm{pH} 2.0$ and $35 \%$ at $\mathrm{pH} 7.4$, which was indicative of obvious pH-responsivity. The MSNs-COOH coated by S5-B/PAA proposed in the present study show potential applications in targeted drug delivery.
\end{abstract}

Keywords: Layer-by-layer, mesoporous silica nanoparticles, poly (glycerol methacrylate), pH-responsivity

\section{Introduction}

Both inorganic nanoparticles and polymer nanocomposites, as well as their assembly methods have received significant attention in the last few decades because of their interesting and well-known properties associated with structural controllability and extremely broad applicability [1-6]. Therefore, they have a strong promise for future applications in drug delivery [7]. For example, mesoporous silica nanoparticles (MSNs) have great properties such as high surface area, high pore volume, prominent biocompatibility, accessible surface functionalization and effective protection for the payloads. As a consequence, MSNs, integrated with ingeniously designed off/on switches for the blockage of nanopore entrances, have been extensively explored as smart drug nanocarriers [8-10]. The drug can

\footnotetext{
${ }^{*}$ Corresponding author: Hong-Yu Zhang, State Key Laboratory of Tribology, Department of Mechanical Engineering, Tsinghua University, Beijing 100084, China. Tel.: +86 010 62773129; Fax: +86 010 62781379; E-mail: zhanghyu@tsinghua.edu.cn.
}

0959-2989/14/\$27.50 @ 2014 - IOS Press and the authors. 
be released under a specific external stimulus. Nanoparticles [11] and polymer brushes [12] have been employed as capping agents to attach to the outer surface of mesoporous materials, preventing the premature release of drug from interior mesopores. Poly (allylamine hydrochloride) (PAH) and sodium salt of poly (styrene sulfonate) (PSSNa) are the most common pH sensitive polymers. He et al. [13] deposited PAH/PSSNa at the outside of ibuprofen-loaded MCM-41, and found that ibuprofen was released under extremely acidic $\mathrm{pH} 1.4$ or high ionic strength. Recently, Sun et al. [14] deposited cucurbit [7] uril (CB [7])-cinnamamide onto Rhodamine B (RhB)-loaded MSNs, and subsequently UV irradiation was used to stimulate the nanocomposites, resulting in trans-to cis-conformational change of cinnamamide, dissociation of the nanocomposites, and finally the release of RhB. Nowadays, relevant research in the field of MSNs-based nanocarriers has focused on the development of new strategies for the off/on switching of nanopore entrances for effective loading and triggered release.

Star-shaped poly (glycerol methacrylate) is distinguished in drug delivery due to its well-defined chemical structure, especially reactive, inexpensive, hydrophilic, biocompatible, and nontoxic properties. In our previous research [15], 1, 4-butanediamine (BDA)-modified linear poly (glycerol methacrylate) (L-B) or BDA-modified star-shaped poly (glycerol methacrylate) (S5-B) and 1, 2ethanediamine (EDA)-modified star-shaped poly (glycerol methacrylate) (S5-E), were found to possess sensitive $\mathrm{pH}$ responsivity. Therefore, integrating them with MSNs was meaningful for $\mathrm{pH}-$ controlled drug delivery. Besides, $\mathrm{RhB}$, as a model cargo with detective ultraviolet absorption band and suitable molecular size, has been widely employed to substitute water-soluble drugs [14]. In nanofabrication, the electrostatic layer-by-layer (LbL) assembly technique has been used to fabricate ultrathin film by precise control of polymer assembly, tailoring the properties of polycation and polyanion by electrostatic deposition on the nanometer scale [16]. However, to our best knowledge, the properties of the nanocomposites combining MSNs, amino-modified poly (glycerol methacrylate) as polycation and $\mathrm{RhB}$ as model cargo, have not been reported in previous research. In the present study, RhB loaded carboxyl-modified MSNs (MSNs-COOH) were prepared and coated with polyelectrolytes through LbL self-assembly, and the loading capacity and the release property of RhB from the nanocomposites were investigated.

\section{Materials and methods}

\subsection{Materials}

Glycidyl methacrylate (GMA), 2-bromoisobutyryl bromide, bipyridyl and $\mathrm{CuBr}$ were purchased from Adamas Reagent Co., Ltd. (Shanghai, China). Polyacrylic acid (PAA) was purchased from Sigma Aldrich Co., Ltd. (Shanghai, China). Cetyltrimethyl ammonium bromide (CTAB), 3(Triethoxysilyl)propyl Isocyanate (ICPTES) were purchased from Aladdin Co., Ltd. (Shanghai, China). 1, 2-Ethanediamine (EDA), tetraethyl orthosilicate (TEOS), RhB and all the other biological reagents and consumable items were from J\&K Reagent Co., Ltd. (Beijing, China).

Amino-modified poly (glycerol methacrylate) was synthesized according to our previously reported procedure $[17,18]$. Briefly, linear PGMA and five-arm PGMA were synthesized from GMA using atom transfer radical polymerization with different initiators, and post-modified with EDA to obtain S5-E and BDA so as to get L-B and S5-B. 


\subsection{Preparation of $\mathrm{MSN}-\mathrm{COOH}$}

CTAB $(1.0 \mathrm{~g})$ and $2 \mathrm{M}$ aqueous $\mathrm{NaOH}$ solution $(3.5 \mathrm{~mL})$ were added to the deionized $\mathrm{H}_{2} \mathrm{O}(480$ $\mathrm{mL})$. The mixture was stirred and heated to $80^{\circ} \mathrm{C}(30 \mathrm{~min})$. Then, TEOS $(5.0 \mathrm{~mL})$ and ICPTES $(0.6$ $\mathrm{mL}$ ) were added via injection sequentially and rapidly. The reaction mixture was heated for another 2 $\mathrm{h}$, and the products were isolated by hot filtration and washed with extensive amount of $\mathrm{H}_{2} \mathrm{O}$ and $\mathrm{MeOH}$. To remove the template surfactants from the mesopores, the as-synthesized silica nanoparticles $(1.0 \mathrm{~g})$ was suspended in acidic $\mathrm{MeOH}$, and then refluxed under $\mathrm{N}_{2}$ for $6 \mathrm{~h}$. The solvent extracted nanoparticles were collected by centrifugation, washed with $\mathrm{MeOH}$, and dried under vacuum to obtain MSNs-COOH [19].

\subsection{Preparation of nanocomposites by LbL self-assembly}

Carboxyl surface functional groups were introduced, and the negative charged silica nanoparticles were achieved. $\mathrm{RhB}$ was dissolved in distilled water with a concentration of $0.5 \mathrm{mmol}$. And $100 \mathrm{mg}$ of MSNs-COOH was ultrasonically dispersed in phosphate buffer saline (PBS) $(10 \mathrm{~mL}, \mathrm{pH}=8.0)$ and mixed with $20 \mathrm{~mL}$ of the $\mathrm{RhB}$ solution. The mixture was stirred at room temperature for $5 \mathrm{~h}$ in the dark. Then $30 \mathrm{mg}$ polycation was added. After stirring for $15 \mathrm{~min}$, the dispersion was centrifuged at $10000 \mathrm{rpm}$ for $3 \mathrm{~min}$ to collect the RhB-loaded MSNs-COOH with S5-B gated nanocomposites. Subsequently, the nanocomposites were re-dispersed in PBS buffer $(\mathrm{pH}=7.4)$ followed by adding PAA solution. The same technique was repeated for another cycle to obtain nanocomposites with four layers' coating. The drug loading capacity (LC) and entrapment efficiency (EE) were calculated against the calibration curve, using the Eqs. (1) and (2), respectively.

$$
\begin{aligned}
& \mathrm{LC}(\%)=\frac{\text { Mass of loaded RhB }}{\text { Mass of RhB-loaded MSNs-COOH }} \\
& \mathrm{EE}(\%)=\frac{\text { Mass of loaded } \mathrm{RhB}}{\text { Initial mass of } \mathrm{RhB}}
\end{aligned}
$$

\subsection{Characterization of nanocomposites}

Transmission electron microscopy (TEM) and scanning electron microscopy (SEM) images were obtained by a JEM-2010F microscope and a Quanta 200 FEG microscope, respectively. To prepare the samples for TEM measurements, one drop of solution containing nanocomposites was placed on carbon-coated copper grid and then dried in air.

Zetasizer Nano ZS173 ${ }^{\circ}$ (Malvern Instruments, MA, USA) was used to detect the Zeta-potential of the MSNs in the coating process, and the results were calculated via the Smoluchowski model fitting of the mobility data. Scattering light was detected at $173^{\circ}$ through a $50 \mu \mathrm{m}$ pin hole at $25^{\circ} \mathrm{C}$. The measurement was conducted in PBS buffer ( $\mathrm{pH}$ 7.4). Each measurement was carried out in triplicate.

Thermo-gravimetric analysis (TGA) experiments were conducted using TA instrument Thermal Analysis (Q5000IR). The samples were heated from $25^{\circ} \mathrm{C}$ to $600^{\circ} \mathrm{C}$ at a heating rate of $10^{\circ} \mathrm{C} / \mathrm{min}$ under argon protection.

Fourier transform infrared spectroscopy (FT-IR) was performed on a nioclet 6700 spectrometer (Thermo Electron, USA) to identify the chemical functional groups in the samples. The substance was 
mixed with $\mathrm{KBr}$ at a ratio of approximately $1 \mathrm{mg}$ sample/100 $\mathrm{mg} \mathrm{KBr}$ to get powder-pressed pellets. Infrared radiation (IR) absorbance data were obtained over a range of wavenumber from $400 \mathrm{~cm}^{-1}$ to $4000 \mathrm{~cm}^{-1}$.

\subsection{Drug release of nanocomposites}

In the experiment, $10 \mathrm{mg}$ freeze-dried RhB-loaded MSNs-COOH-(S5-B/PAA) 2 nanocomposites were dispersed into $1 \mathrm{~mL}$ of PBS buffer $(\mathrm{pH}=7.4)$, acetic acid $(\mathrm{pH}=4.0)$ and phosphoric acid buffer $(\mathrm{pH}=2.0)$, respectively. Then the solution with nanocomposites was put into dialysis bag (cut-off $\mathrm{MW}=6000 \sim 8000)$, and finally immersed into the release mediums $(20 \mathrm{~mL})$, separately. At certain time intervals, $4 \mathrm{~mL}$ release medium was withdrawn and replaced with fresh PBS buffer. RhB released from the complex was determined by UV-vis spectroscopy at a wavelength of $552 \mathrm{~nm}$ at $\mathrm{pH}$ values of 7.4, 4.0, and 2.0, respectively. All release tests were run in triplicate, and the error bar in the plot was standard deviation.

\section{Results and discussion}

\subsection{LC and EE at different $p H$ values}

$\mathrm{PH}=8.0$ and $\mathrm{pH}=5.0$ was used to investigate the impact of $\mathrm{pH}$ on the loading capacity (LC) and entrapment efficiency (EE) of different polyelectrolytes. The tests were repeated for three times and the results were shown in Table 1. Obviously, both the LC and EE were higher at $\mathrm{pH}=8.0$ than those at $\mathrm{pH}=5.0$. This may be attributed to the fact that carboxyl groups on MSNs-COOH become more electron-rich at $\mathrm{pH}=8.0$, which give easy access for $\mathrm{N}^{+}$on $\mathrm{RhB}$ to adhering to MSNs-COOH by electrostatic interaction. Meanwhile, in the coating process, $\mathrm{pH} 7.4$ was more proficient for polyelectrolytes to form electrostatic interaction. Besides, when the polycation was S5-B, both the LC and $\mathrm{EE}$ were higher than other polycations. A reasonable assumption can be due to the fact that S5-B has a big molecular structure, and the size was appropriate for blocking the mesoporous on MSNs$\mathrm{COOH}$. Therefore, the further research focused on S5-B type.

\subsection{Morphology characterization}

The morphology of MSNs-COOH nanoparticles and the RhB-loaded and S5-B/PAA-coated nanocomposites were investigated by TEM and SEM. TEM image of MSNs-COOH, as shown in Figure 1(a), demonstrated that the average diameter of the resulting spherical-shaped nanoparticles

Table 1

Comparison of the mean value of $\mathrm{LC}$ and $\mathrm{EE}$ for $\mathrm{RhB}$ loaded MSNs-COOH at $\mathrm{pH}=8$ and $\mathrm{pH}=5$

\begin{tabular}{lllll}
\hline \multirow{2}{*}{4 layers } & $\mathrm{pH}=8.0$ & & $\mathrm{pH}=5.0$ & \\
\cline { 2 - 5 } & $\mathrm{LC}(\%)$ & $\mathrm{EE}(\%)$ & $\mathrm{LC}(\%)$ & EE (\%) \\
S5-E & 9.57 & 44.1 & 2.7 & 17.3 \\
S5-B & 10.1 & 44.4 & 3.0 & 19.2 \\
L-B & 6.6 & 41.3 & 1.6 & 10.3 \\
\hline
\end{tabular}


was about $100 \mathrm{~nm}$, and the internal mesoporous could be clearly observed. After RhB loading and LbL coating with S5-B/PAA, the average diameter increased to $180 \mathrm{~nm}$ approximately, as shown by the TEM image in Figure 1(b), and the internal mesoporous disappeared. This suggested that RhB was successfully loaded. In addition, coating the polyelectrolytes resulted in a much rougher surface, as shown by the SEM image of the nanocomposites in Figure 1(c).

Zeta-potentials have been shown as an excellent indicator of multi-layer film growth on particles. The potential of MSNs-COOH was $-23 \mathrm{mV}$, and it changed to $-6 \mathrm{mV}$ after loading of $\mathrm{RhB}$, as displayed in Figure 2. In the coating process, the potentials fluctuated between $+20 \mathrm{mV}$ and $-25 \mathrm{mV}$, which was in accordance with the electrostatic principle. This result proved that polyelectrolytes were successfully coated on MSNs-COOH.

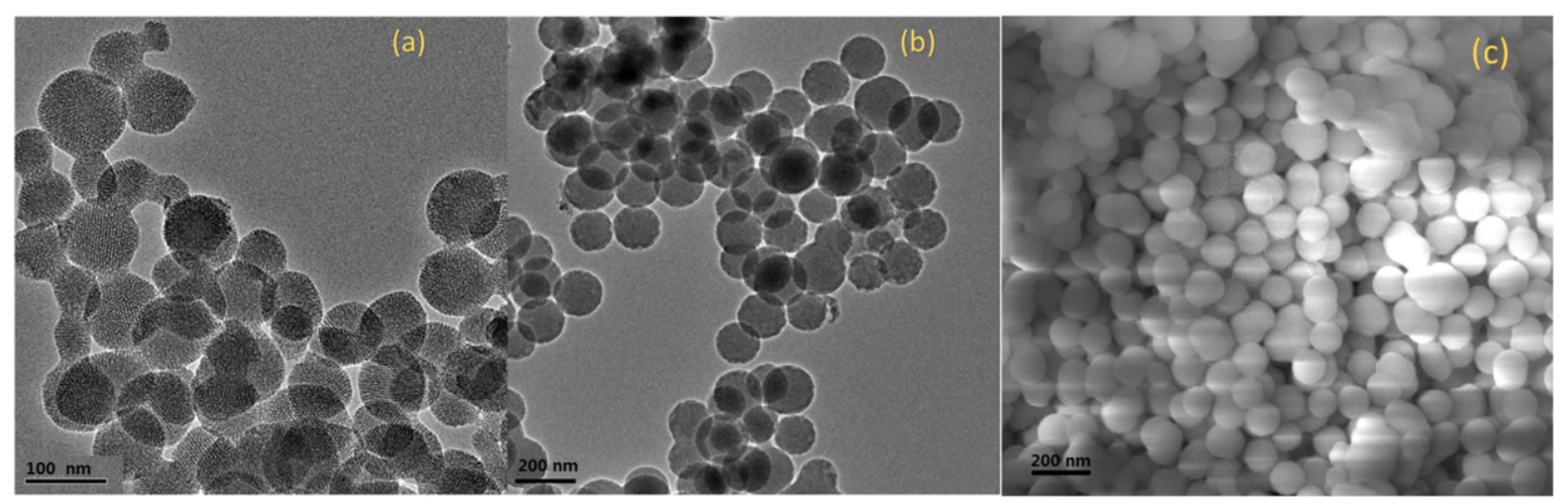

Fig. 1. (a) TEM image of MSNs-COOH; (b and c) TEM and SEM images of RhB loaded MSNs-COOH with four layers of S5-B/PAA, respectively.

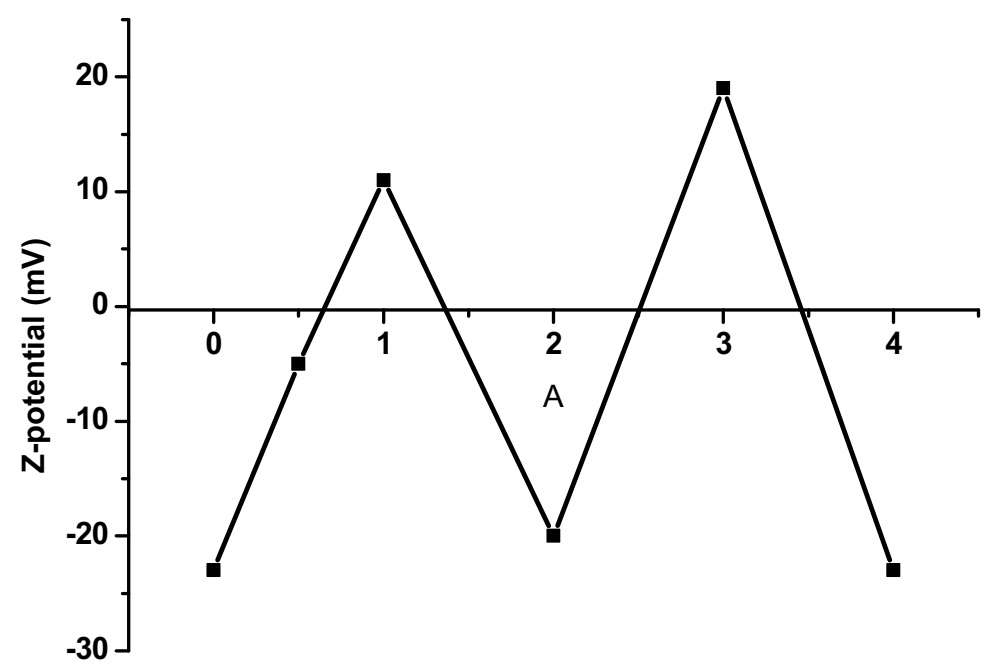

Fig. 2. The variation of Zeta-potential during the coating process of S5-B/PAA. 


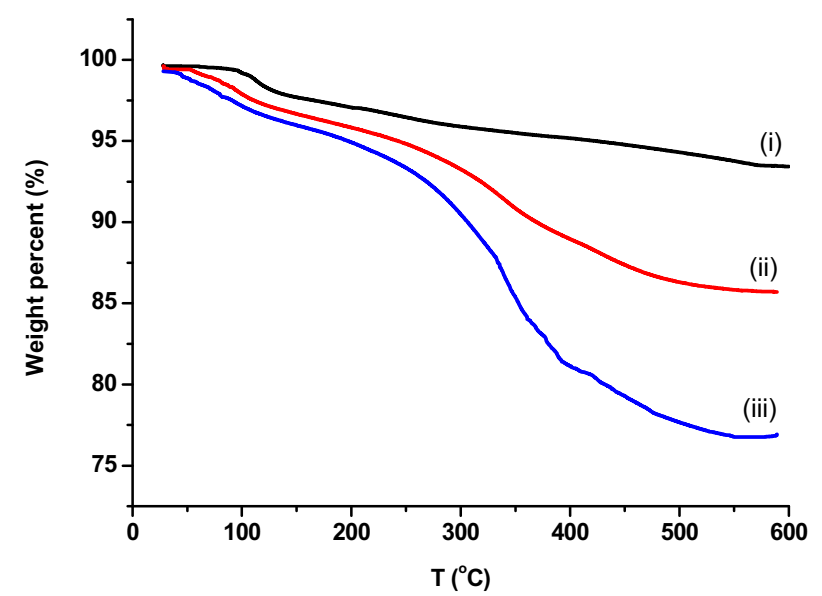

Fig. 3. TGA curves for (i) MSNs-COOH; (ii) MSNs-COOH-RhB; (iii) MSNs-COOH-RhB-(S5-B/PAA) 2 .

Quantification of the entrapped RhB and the polyelectrolyte coating was conducted by TGA, as indicated by Figure 3. Based on this figure, about $8 \%$ of $\mathrm{RhB}$ was loaded, and $9 \%$ of polyelectrolytes was coated. For MSNs-COOH, the weight loss between $120^{\circ} \mathrm{C}$ and $600^{\circ} \mathrm{C}$ was about $7 \%$ (i), corresponding to the decomposition of carboxyl groups of the MSNs-COOH. The weight loss increased to $15 \%$ for RhB-loaded MSNs- $\mathrm{COOH}$ (ii), indicating that about $8 \%$ of $\mathrm{RhB}$ was loaded into MSNs-COOH. The result was a little lower than the loading capacity. After LbL self-assembly, the weight percentage dropped to $76 \%$, indicating that the weight percentage of polyelectrolyte coating is $9 \%$ (iii). These results further confirmed that $\mathrm{RhB}$ was loaded into MSNs- $\mathrm{COOH}$ and polyelectrolytes were coated on MSNs-COOH successfully.

FT-IR spectra of MSNs-COOH before and after loading of RhB were demonstrated in Figure 4. The characteristic peaks at, $794 \mathrm{~cm}^{-1}, 960 \mathrm{~cm}^{-1}$ and $1106 \mathrm{~cm}^{-1}$ are attributed to Si-O-Si symmetric

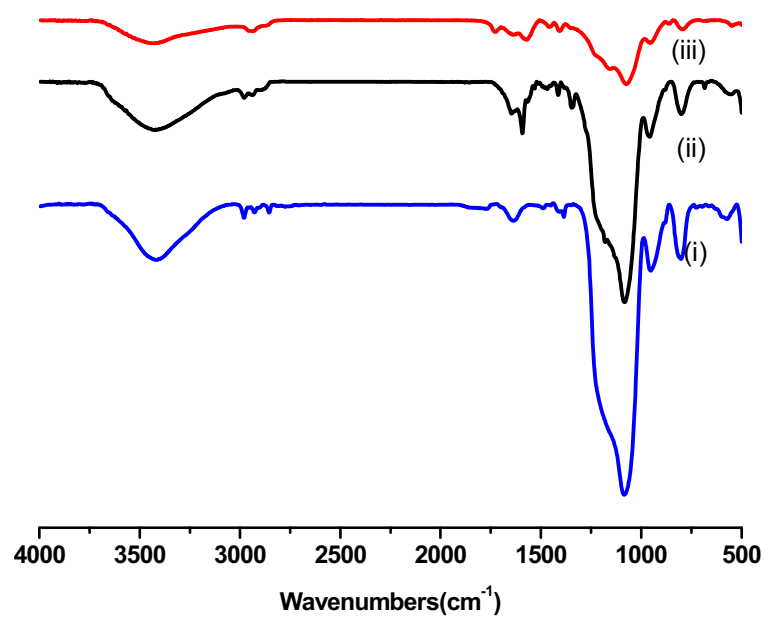

Fig. 4. FT-IR spectra of (i) MSNs-COOH; (ii) MSNs-COOH-RhB; (iii) MSNs-COOH-RhB-(S5-B/PAA) 2 . 


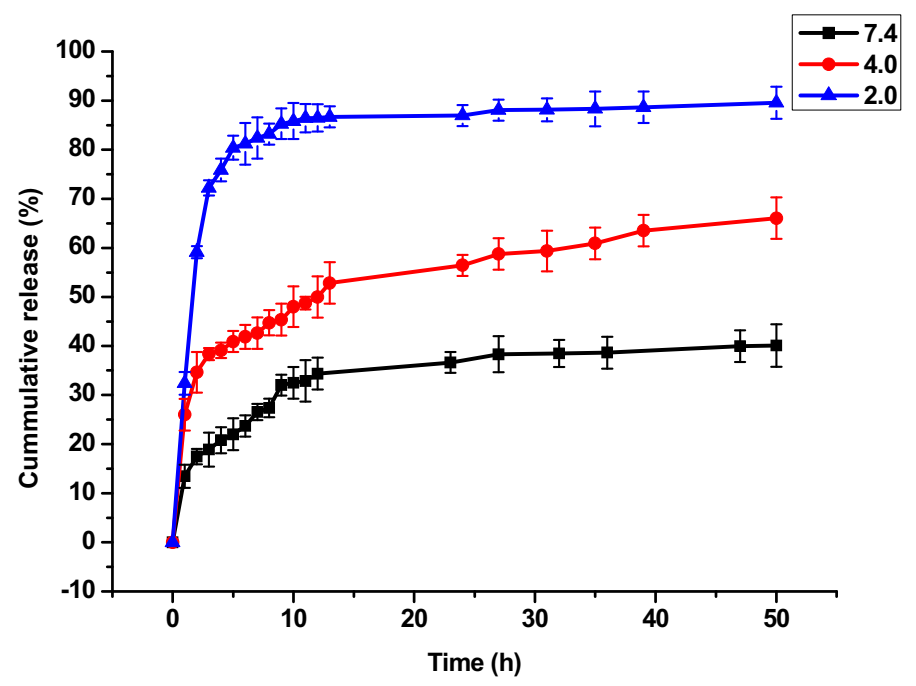

Fig. 5. RhB release from MSNs-COOH-RhB-(S5-B/PAA) $)_{2}$ at $\mathrm{pH}=7.4,4.0$, and 2.0.

stretching, external Si-OH asymmetric stretching, and Si-O-Si asymmetric stretching, respectively. The peaks at $3480 \mathrm{~cm}^{-1}$ and $1652 \mathrm{~cm}^{-1}$ corresponded to the $-\mathrm{OH}$ stretching from the water molecules retained by MSNs-COOH and MSNs-COOH itself, respectively. After the loading process, new bands at $1360 \mathrm{~cm}^{-1}$ (aromatic C-N stretching vibration), $1582 \mathrm{~cm}^{-1}$ (vibration modes of benzene ring) demonstrated that $\mathrm{RhB}$ was successfully loaded into MSNs-COOH (ii). For polyelectrolyte coating, the absorption bands appeared at $1710 \mathrm{~cm}^{-1}$ and $1637 \mathrm{~cm}^{-1}$ are attributed to the $\mathrm{C}=\mathrm{O}$ from PAA and $\mathrm{NH}_{2}$ from amino-modified PGMA, respectively (iii).

Figure 5 showed the controlled release property of $\mathrm{RhB}$ in PBS solution. The delivery system of nanocomposites was released about $35 \%$ in the PBS solution of $\mathrm{pH} 7.4$. While, the plateau of $\mathrm{pH}=4.0$ and $\mathrm{pH}=2.0$ reached $65 \%$ and $90 \%$, respectively. The release rate increased with the decrease of the $\mathrm{pH}$ value of the solution. This may be due to the adjustable permeability of S5-B/PAA polyelectrolytes coated on the MSNs at different $\mathrm{pH}$ values. Besides, $\mathrm{RhB}$ exhibited burst release within $5 \mathrm{~h}$, and this may also be attributed to the fact that the lower the $\mathrm{pH}$ of the medium, the less the negative charges on the surface of the MSNs-COOH. Accordingly, weakening the electrostatic interaction promoted release of $\mathrm{RhB}$. Therefore, an apparent $\mathrm{pH}$-controlled release of $\mathrm{RhB}$ was achieved using the present delivery system.

\section{Conclusion}

In the present study, the nanocomposites composed of RhB-loaded MSNs-COOH and S5-E/PAA, S5-B/PAA and L-B/PAA coatings are obtained by LbL self-assembly. Among the three kinds of polycations, S5-B shows the highest loading capacity and entrapment efficiency. By means of adjusting the $\mathrm{pH}$ value of PBS buffer, the cumulative release of RhB from MSNs-COOH shows a low leakage at $\mathrm{pH} 7.4$ after $13 \mathrm{~h}$, while the value significantly increases to $65 \%$ at $\mathrm{pH} 4.0$ and to $95 \%$ at $\mathrm{pH}$ 2.0. These results demonstrate that the nanocomposites are $\mathrm{pH}$-responsive, implying that this biocompatible system could be potentially applied as nanocarrier in targeted drug delivery. 


\section{Acknowledgement}

This project is supported by National Natural Science Foundation of China (Grant No. 51375008, 51005130), and the Research Fund of State Key Laboratory of Tribology, Tsinghua University, China (Grant No. SKLT12B06).

\section{References}

[1] Y.J. Wang and F. Caruso, Mesoporous silica spheres as supports for enzyme immobilization and encapsulation, Chem. Mater. 17 (2005), 953-961.

[2] X.J. Wan, G.Y. Zhang and S.Y. Liu, pH-disintegrable polyelectrolyte multilayer-coated mesoporous silica nanoparticles exhibiting triggered co-release of cisplatin and model drug molecules, Macromol. Rapid Commun. 32 (2011), 10821089.

[3] J.L. Vivero-Escoto, I.I. Slowing, B.G. Trewyn and V.S.-Y. Lin, Mesoporous silica nanoparticles for intracellular controlled drug delivery, Small 6 (2010), 1952-1967.

[4] S.H. Wu, Y. Hung and C.Y. Mou, Mesoporous silica nanoparticles as nanocarriers, Chem. Commun. 47 (2011), 99729985.

[5] Z.X. Li, J.C. Barnes, A. Bosoy, J.F. Stoddart and J.I. Zink, Mesoporous silica nanoparticles in biomedical applications, Chem. Soc. Rev. 41 (2012), 2590-2605.

[6] L. Pan, Q. He, J. Liu, Y. Chen, M. Ma, L.L. Zhang and J. Shi, Nuclear-targeted drug delivery of TAT peptideconjugated monodisperse mesoporous silica nanoparticles, J. Am. Chem. Soc. 134 (2012), 5722-5725.

[7] L.M. Bronstein and Z.B. Shifrina, Dendrimers as encapsulating, stabilizing, or directing agents for inorganic nanoparticles, Chem. Rev. 111 (2011), 5301-5344.

[8] F. Balas, M. Manzano, P. Horcajada and M. Vallet-Regi, Confinement and controlled release of bisphosphonates on ordered mesoporous silica-based materials, J. Am. Chem. Soc. 128 (2006), 8116-8117.

[9] M. Vallet-Regi, F. Balas and D. Arcos, Mesoporous materials for drug delivery, Angew. Chem. Int. Ed. 46 (2007), $7548-7558$

[10] M. Vallet-Regí and E. Ruiz-Hernández, Bioceramics: From bone regeneration to cancer nanomedicine, Adv. Mater. 23 (2011), 5177-5218.

[11] C.Y. Lai, B.J. Trewyn, D.M. Jeftinija, K. Jeftinija, S. Xu, S. Jeftinija and V.S-Y. Lin, A mesoporous silica nanospherebased carrier system with chemically removable CdS nanoparticle caps for stimuli-responsive controlled release of neurotransmitters and drug molecules, J. Am. Chem. Soc. 125 (2003), 4451-4459.

[12] D.R. Radu, C.Y. Lai, K. Jeftinija, E.W. Rowe, S. Jeftinija and V.S.-Y. Lin, A polyamidoamine dendrimer-capped mesoporous silica nanosphere-based gene transfection reagent, J. Am. Chem. Soc. 126 (2004), 13216-13217.

[13] Q. He and J. Shi, Mesoporous silica nanoparticle based nano drug delivery systems: synthesis, controlled drug release and delivery, pharmacokinetics and biocompatibility, J. Mater. Chem. 21 (2011), 5845-5855.

[14] Y.L. Sun, B.J. Yang, S.X.-A. Zhang and Y.W. Yang, Cucurbituril pseudorotaxane-based photoresponsive supramolecular nanovalve, Chem. Eur. J. 18 (2012), 92125-9216.

[15] K. Ariga, Y.M. Lvovc, K. Kawakamia, Q. Jia and J.P. Hill, Layer-by-layer self-assembled shells for drug delivery, Adv. Drug Deliver Rev. 63 (2011), 762-771.

[16] H. Gao, M.C. Jones, P. Tewari, M. Ranger and J.C. Leroux, Document star-shaped alkylated poly (glycerol methacrylate) reverse micelles: synthesis and evaluation of their solubilizing properties in dichloromethane, J. Polym. Sci. Part A: Polym Chem 45 (2007), 2425-2435.

[17] X.Y. Lu, H. Gao, C. Li, Y.W. Yang, Y.N. Wang, Y.G. Fan, G.L. Wu and J.B. Ma, Document polyelectrolyte complex nanoparticles of amino poly(glycerol methacrylate)s and insulin, Int. J. Pharm. 423 (2012), 195-201.

[18] Y.F. Sun, Y.L. Sun, L.Z. Wang, J.B. Ma, Y.W. Yang and H. Gao, Nanoassembles constructed from mesoporous silica nanoparticles and surface-coated multilayer polyelectrolytes for controlled drug delivery, Micropor. Mesopor. Mat. 184 (2014), 245-253.

[19] Y.F. Sun, H Gao, Y.W. Yang, A. Wang, G. Wu, Y. Wang, Y. Fan and J.B. Ma, Layer-by-layer supramolecular assemblies based on linear and star-shaped poly (glycerol methacrylate)s for doxorubicin delivery, J. Biomed. Mater. Res. Part A. 101 (2013), 2164-2173. 